\title{
Quantitatively Correct UV-vis Spectrum of Ferrocene with TDB3LYP
}

\author{
Ulrike Salzner* \\ Department of Chemistry, Bilkent University, 06800 Bilkent, Ankara, Turkey
}

ABSTRACT: The ultraviolet-visible light (UV-vis) absorption spectrum of ferrocene is modeled with time-dependent density functional theory employing LSDA, BLYP, B3LYP, and CAM-B3LYP functionals in combination with 6-31G*, 6$31+\mathrm{G}^{*}$, CC-PVTZ, and aug-CC-PVTZ basis sets. With the exception of LSDA, all functionals predict a reasonable Fe-CP distance of $\sim 1.67 \AA$. Diffuse functions are essential for the

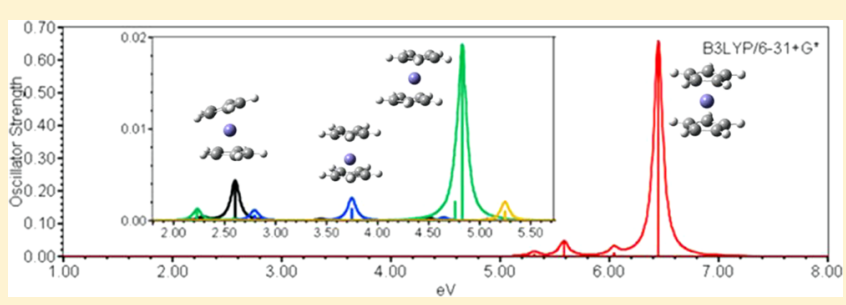
strongly allowed states at high energy but of lesser consequence for the visible range of the spectrum. Dipole forbidden states are examined with vibrationally excited structures, obtained from the normal modes of the infrared (IR) spectrum. Despite earlier claims, TDB3LYP predicts the UV-vis spectrum of ferrocene quantitatively correct. TDBLYP predicts a large number of spurious charge-transfer states, TDCAM-B3LYP and TDwB97XD are correct in the low-energy region but overestimate the energy of strongest peak of the spectrum by $0.8 \mathrm{eV}$. The amount of charge transfer involved in " $\mathrm{d}-\mathrm{d}$ transitions" is equal to that in "charge-transfer states".

\section{INTRODUCTION}

Ferrocene was discovered in $1951^{1}$ and has revolutionized the views of chemists about how metals bind to organic $\pi$-systems. Its electronic structure and ultraviolet-visible light (UV-vis) absorption spectrum have become textbook ${ }^{2}$ examples, and one would assume that not much new can be said about them. However, comparison between results obtained with timedependent density functional theory (TDDFT) by the author and theoretical data from the literature ${ }^{3-15}$ revealed several discrepancies, which prompted the present investigation.

Pure ferrocene is a very stable light orange powder, and the UV-vis absorption spectrum of ferrocene has been investigated in detail. ${ }^{16-19}$ Upon cooling, the color changes from orange to lemon yellow. ${ }^{16,17}$ The orange color stems from a dipoleforbidden absorption at $440 \mathrm{~nm}(2.81 \mathrm{eV})$ and a shoulder at $528 \mathrm{~nm}(2.34 \mathrm{eV}),{ }^{16-18}$ both of which gain oscillator strength only through vibronic coupling. ${ }^{8}$ Outside the visible range, a weak peak occurs at $324 \mathrm{~nm}(3.83 \mathrm{eV})$ and several relatively weak but allowed absorptions are present between $265 \mathrm{~nm}$ and $230 \mathrm{~nm}$. The strongest absorption of ferrocene is observed at $202 \mathrm{~nm}(6.12 \mathrm{eV})$ in solution and $6.31 \mathrm{eV}$ in the vapor phase. $^{16-19}$ The oscillator strength of this allowed absorption is 350 times stronger than that of the peak at $440 \mathrm{~nm}$.

B3LYP produces the structural parameters of ferrocene with errors of $2 \mathrm{pm}$ or less, ${ }^{20}$ and there is consensus that, in agreement with experiment, theory predicts the eclipsed form to be more stable than the staggered one. Several authors have cautioned, however, against using B3LYP for excited-state calculations of ferrocene. ${ }^{6,10,12,15}$ Boulet et al., ${ }^{6} \mathrm{Li}$ et al., ${ }^{10}$ and Scuppa et al. ${ }^{12}$ promoted gradient-corrected pure DFT functionals, and Fromager et al. ${ }^{15}$ promoted the rangeseparated CAM-B3LYP functional. In this investigation, TDDFT results with several functionals and different allelectron basis sets are analyzed. It is demonstrated that B3LYP works perfectly fine and that the differences between B3LYP results and experiment found in earlier studies arise from incorrect assignment of the excited states, ${ }^{6,10,12,15}$ use of inadequate pseudopotentials, ${ }^{6}$ and that agreement between theory and experiment at other levels of theory was manipulated by using $\mathrm{Cp}-\mathrm{Fe}$ distances that were too short. ${ }^{6,12}$

Based on ligand field theory, the low-energy peaks are assigned to be $\mathrm{d}-\mathrm{d}$ transitions and the stronger absorptions to charge-transfer (CT) states. ${ }^{15,21}$ Analysis of density differences and charge differences on Fe between ground and excited states reveals that low- and high-lying excited states involve similar amounts of charge transfer, so that the distinction between $n$ $\mathrm{d}-\mathrm{d}$ and CT transitions ${ }^{21}$ is not justified.

The paper is outlined as follows. First, the effects of structure, solvent, theoretical method, and basis set are evaluated for the high-energy part of the spectrum. Second, vibrationally excited structures of ferrocene are obtained from frequency calculations and UV-vis spectra are predicted for distorted conformers. This allows identifying the peaks that become visible only upon vibration. Then the area between 4 and $6 \mathrm{eV}$, which includes the onset of allowed transitions, is investigated. Finally, the electronic transitions are characterized with density difference plots and natural population analysis. ${ }^{22}$

\section{METHODS}

The structure of ferrocene was optimized in eclipsed (D5h) and staggered (D5d) conformations. UV-vis spectra were calculated for both structures and for several lower symmetry forms (Scheme 1) that were obtained from the first seven vibrational modes of D5h ferrocene at the B3LYP/6-31+G* level of theory.

UV-vis spectra were calculated with TDDFT. The number of excited states was increased until the strongly allowed excited

Received: April 19, 2013

Published: July 23, 2013 
Scheme 1

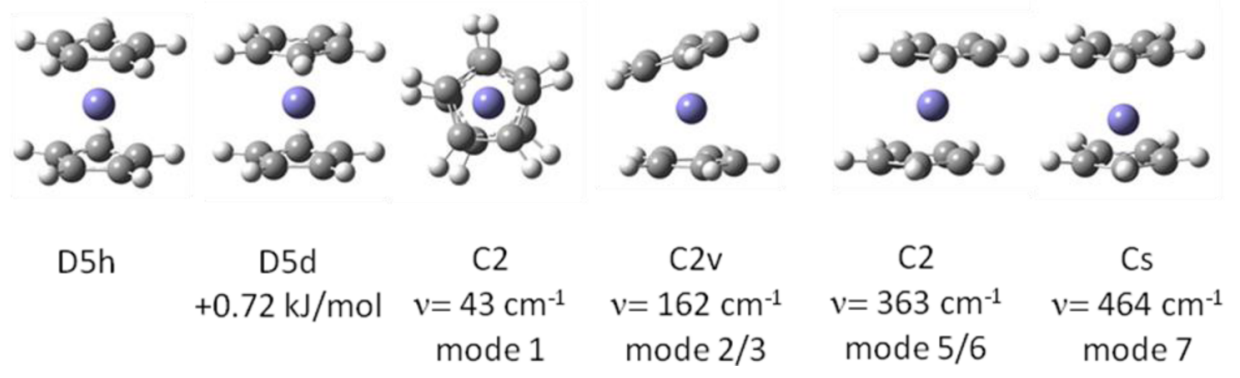

state that occurs experimentally at $6.31 \mathrm{eV}$ with oscillator strength 0.69 in the vapor phase was found. The number of excited states required to reach the strongly allowed state depends on the level of theory and basis set and lies between 36 and 90. The functionals tested include LSDA, ${ }^{23,24} \mathrm{BLYP}^{25,26}$ B3LYP, ${ }^{26,27}$ CAM-B3LYP, ${ }^{28}$ and wB97XD. ${ }^{29}$ The basis sets employed are $6-31 G^{*}, 6-31+G^{*}, 6-311+G^{*}$, CC-PVTZ, and aug-CC-PVTZ. ${ }^{30,31}$ Relativistic effects were not considered because comparison with results of Scuppa et al. ${ }^{12}$ and Fromager et al., ${ }^{15}$ who employed relativistic pseudo-potentials, revealed no major differences. Solvent effects of methylene dichloride were treated with the polarized continuum method. ${ }^{32}$ All calculations are done with Gaussian 09. ${ }^{33} \mathrm{UV}$ spectra are generated with Gabedit. ${ }^{34}$ The peaks are convoluted with Lorentzian functions with peak widths at half-maximum of $0.05 \mathrm{eV}$. Orbitals and density differences are plotted with Gauss View 5 .

\section{RESULTS}

Effect of Structure and Solvent on the Spectrum. The eclipsed D5h ferrocene is $0.71 \mathrm{kcal} / \mathrm{mol}$ more stable than the staggered D5d form at B3LYP/6-31G* and B3LYP/6-31+G*. The first vibrational mode, which rotates the cyclopentadienyl (Cp) rings relative to each other, has a vibrational energy of 43 $\mathrm{cm}^{-1}$ at B3LYP/6-31+G*. Because of the small energy difference between both forms and because of the low vibrational energy, both forms are expected to be present at room temperature.

The distance between $\mathrm{Fe}$ and the $\mathrm{Cp}$ rings in the $\mathrm{D} 5 \mathrm{~h}$ structure is $1.654 \AA$ at $\mathrm{B} 3 \mathrm{LYP} / 6-31 \mathrm{G}^{*}, 1.675 \AA$ at $\mathrm{B} 3 \mathrm{LYP} / 6$ $31+\mathrm{G}^{*}$, and $1.690 \AA$ at B3LYP/aug-CC-PVTZ, showing that increasing the basis set increases the $\mathrm{Fe}-\mathrm{Cp}$ distance. With the $6-31+\mathrm{G}^{*}$ basis set, the $\mathrm{Fe}-\mathrm{Cp}$ distance is $1.675 \AA$ with BLYP and B3LYP, $1.660 \AA$ with CAM-B3LYP/6-31+G*, and $1.645 \AA$ with wB97XD, indicating that increasing amount of HartreeFock (HF) exchange decreases the $\mathrm{Fe}-\mathrm{Cp}$ distance. These values are in reasonable agreement with coupled cluster theory $(1.655 \AA)$ and experiment $(1.661 \AA) .{ }^{5,20}$ LSDA/6-31+G* predicts a $\mathrm{Fe}-\mathrm{Cp}$ distance of $1.579 \AA$ that is too short.

Figure 1 compares gas and solution phase absorption spectra of D5h and D5d ferrocene at B3LYP/6-31+G*. There are no allowed absorptions in the visible range for both forms. The first peak with low oscillator strengths occurs at $5.32 \mathrm{eV}$ in solution and at $5.59 \mathrm{eV}$ in the gas phase. Solvent effects as predicted with the polarized continuum model reduce the excitation energy of the main peak by $0.07 \mathrm{eV}$ for D $5 \mathrm{~h}(0.09 \mathrm{eV}$ for the D5d), which is about half of the experimental solvent effect $(0.11 \mathrm{eV}-0.19 \mathrm{eV})$. The solvent effect on the oscillator strength is exaggerated. Theory predicts increase of 0.41 for both forms but the experimental solvent effect on the oscillator

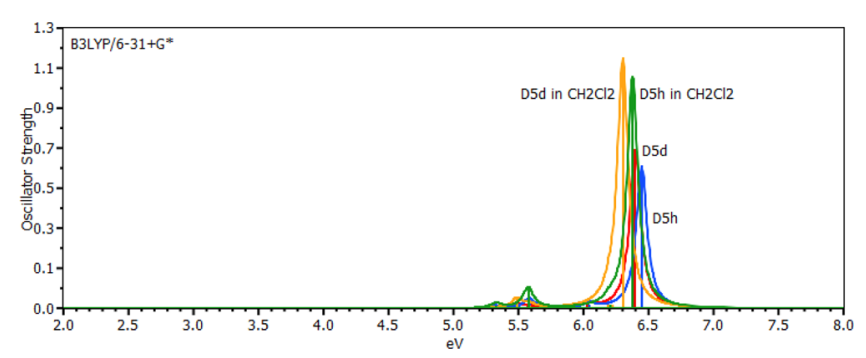

Figure 1. UV spectra of gas phase and methylene dichloride solution spectra of eclipsed (D5h) and staggered (D5d) ferrocene at the B3LYP/6-31+G* level of theory.

strength is only $0.08 .^{18}$ It will be shown, throughout the paper, that oscillator strength is also very sensitive to details of the basis set.

The main peak is observed for D5h ferrocene at $6.45 \mathrm{eV}$ (gas) and $6.38 \mathrm{eV}$ (in $\mathrm{CH}_{2} \mathrm{Cl}_{2}$ ), and for $\mathrm{D} 5 \mathrm{~d}$ at $6.40 \mathrm{eV}$ (gas) and $6.31 \mathrm{eV}$ (in $\mathrm{CH}_{2} \mathrm{Cl}_{2}$ ). The experimental values are $6.31 \mathrm{eV}$ in the vapor phase and $6.12 \mathrm{eV}^{18}$ or $6.20^{19}$ in solution. The oscillator strengths are larger by 0.08 for the D5d form in gas and solution phases. Hence, any presence of D5d ferrocene may cause peak broadenings or appear as shoulders in experimental spectra. The D5d form will not be considered further.

Effect of Basis Set Enlargement. The effect of basis set enlargement at the B3LYP and CAM-B3LYP levels is seen in Figure 2. Data with basis sets that include diffuse functions are summarized in Table 1. B3LYP/aug-CC-PVTZ was taken as the reference for a tentative assignment of the allowed states to
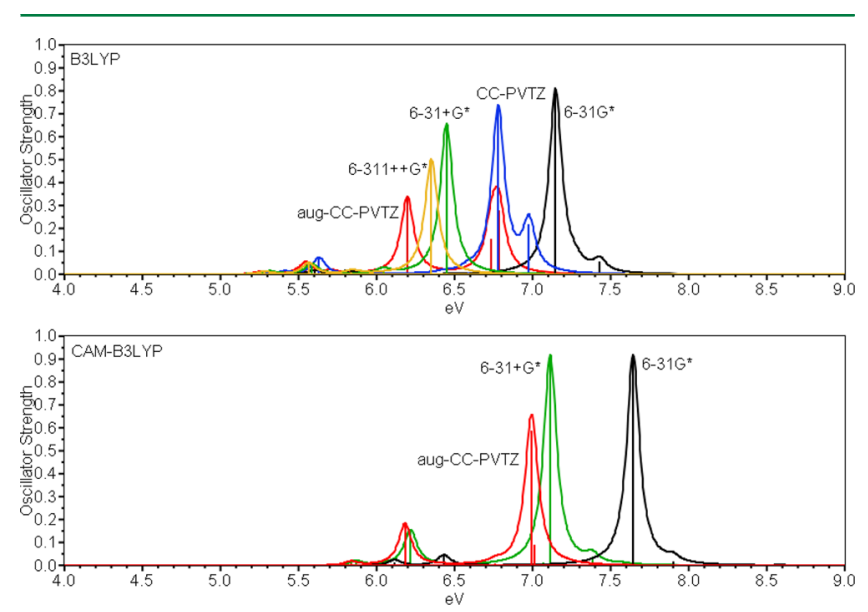

Figure 2. Effect of basis set enlargement on the UV spectrum of eclipsed (D5h) ferrocene with the B3LYP and CAM-B3LYP functionals. 


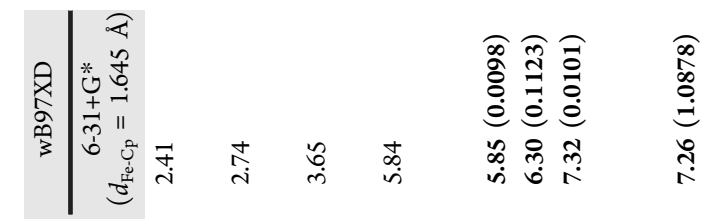

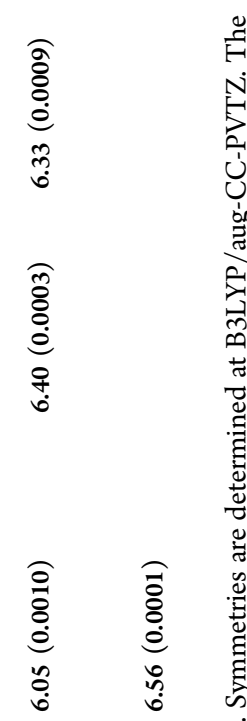

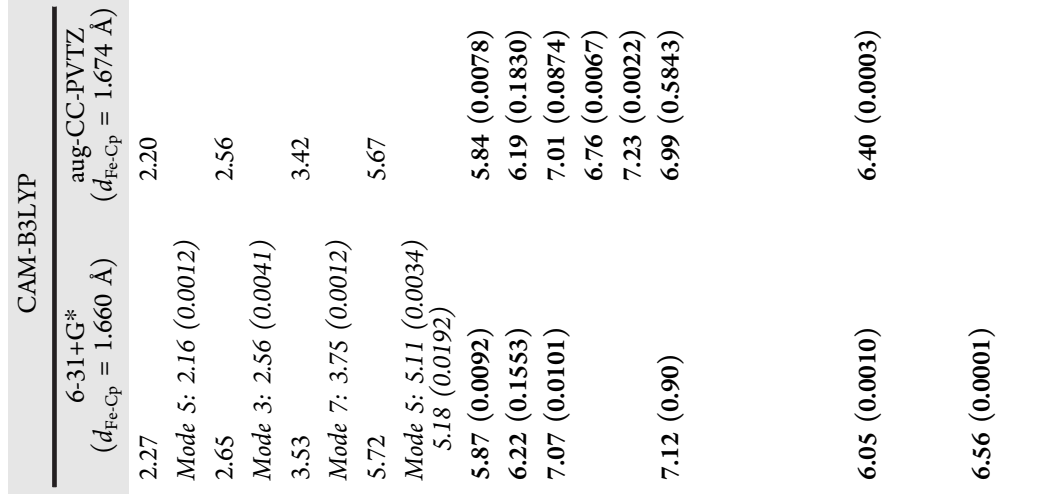

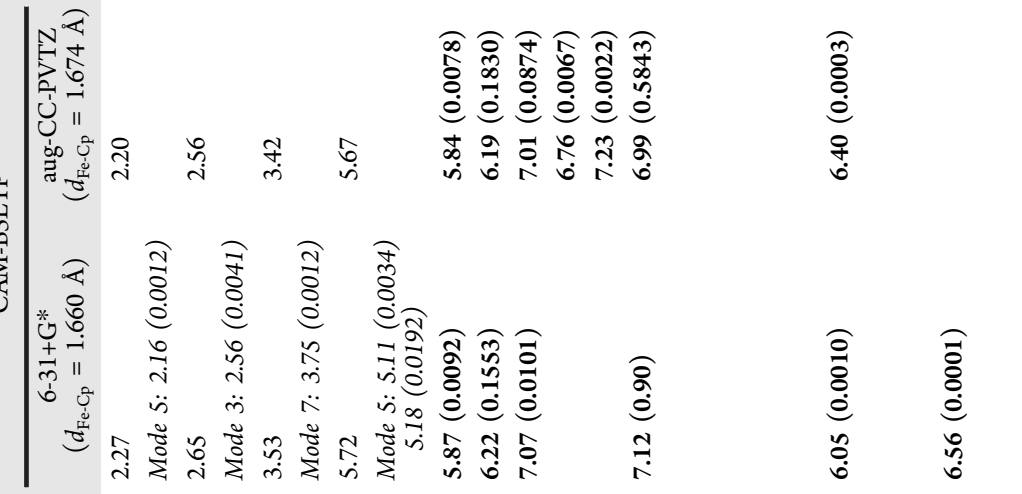

$\nsubseteq$

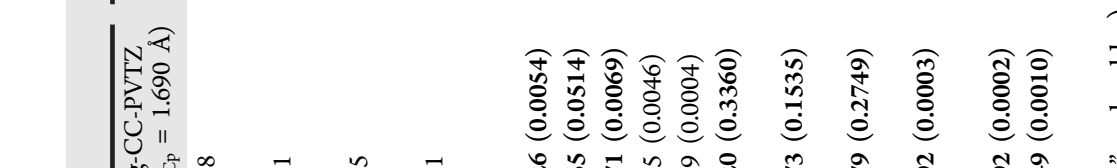

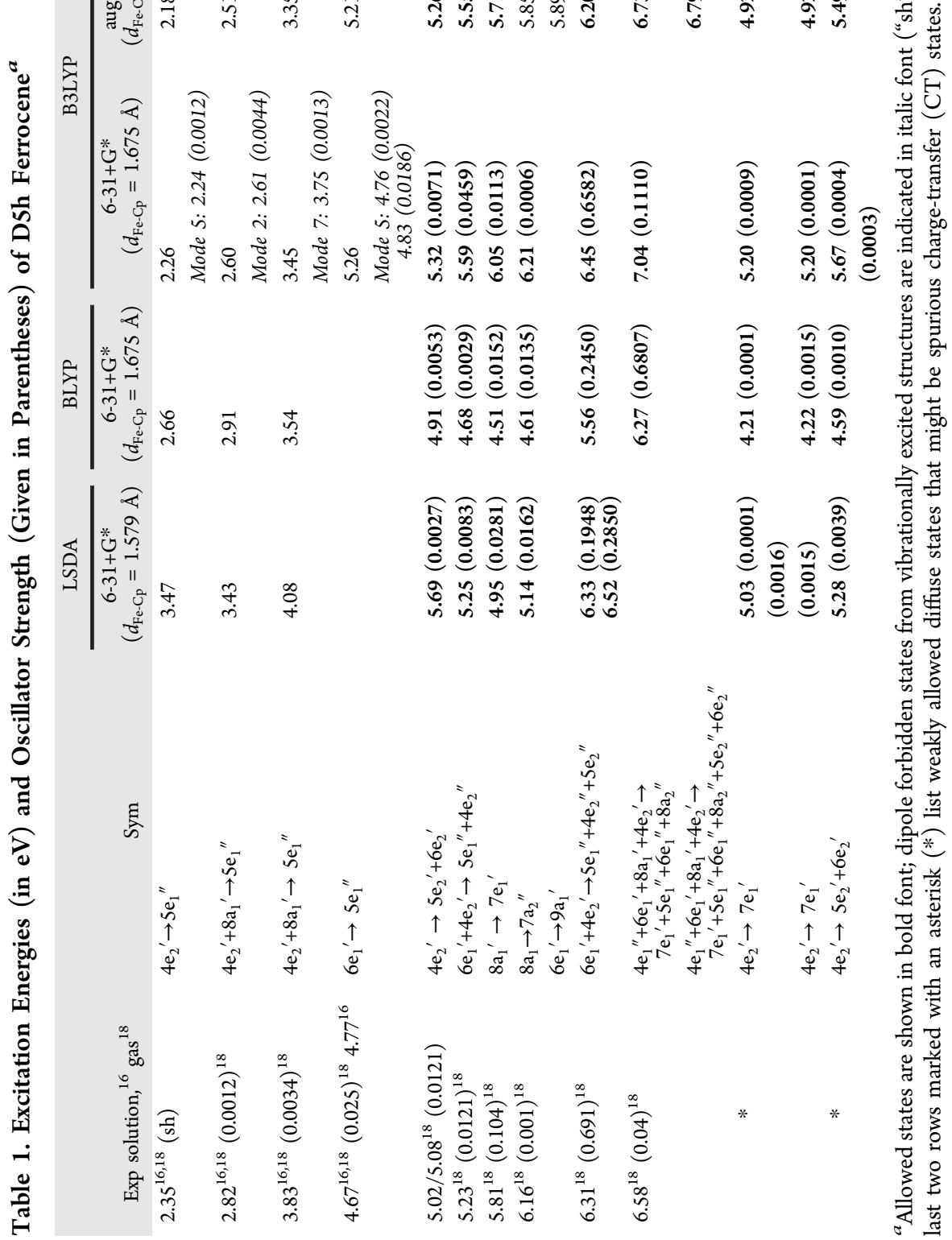


experiment. States at the other levels of theory were organized so that their electronic configurations match those at B3LYP/ aug-CC-PVTZ and are therefore not listed according to increasing energies.

Basis set enlargement lowers the excitation energy of the strong peak. Most important are diffuse functions as they lower the excitation energy by 0.7 , from $6-31 G^{*}$ to $6-31+G^{*}$ and by $0.6 \mathrm{eV}$ upon augmentation of the CC-PVTZ basis set. Increasing the basis set from $6-31+\mathrm{G}^{*}$ to aug-CC-PVTZ reduces the energy of the strong peak by another $0.25 \mathrm{eV}$. The lower energy peaks respond very little to the presence of diffuse functions and differ by only up to $0.1 \mathrm{eV}$ with the $6-31+\mathrm{G}^{*}$ and aug-CC-PVTZ basis sets, so that $6-31+\mathrm{G}^{*}$ is adequate for the lower energy range of the spectrum.

Increasing the basis set reduces the oscillator strength of the main peak. At B3LYP/aug-CC-PVTZ, the oscillator strength is less than half of the experimental one. The two higher peaks at 6.73 and $6.79 \mathrm{eV}$ arise from similar electron configurations as the main peak and their presence therefore reduces the oscillator strength of the main peak..$^{35-37}$ The transfer of oscillator strength into these states at the ionization threshold of ferrocene at $6.6-7.1 \mathrm{eV}^{38}$ might be an artifact of the diffuse but finite basis set.

Compared to B3LYP, the allowed states are shifted to higher energy by $0.8 \mathrm{eV}$ with the range-separated CAM-B3LYP functional employing the same basis set. The dipole-forbidden low-energy states differ by only up to $0.1 \mathrm{eV}$. This trend seems logical, as range-separated functionals increase the amount of $\mathrm{HF}$ exchange as a function of the distance from the center. Therefore, high-energy diffuse excited states should be affected more than compact valence excited states. Compared to the experiment, CAM-B3LYP/aug-CC-PVTZ overestimates the main peak by $0.7 \mathrm{eV}$. The basis set dependence is less with CAM-B3LYP than with B3LYP, and there is no splitting of the main peak into several components. This might be attributed to the larger energy difference between orbital energies with increasing amount of HF exchange, which reduces mixing of different electronic configurations.

Effect of the Density Functional. Spectra predicted with the $6-31+G^{*}$ basis set are shown in Figure 3. The results for the

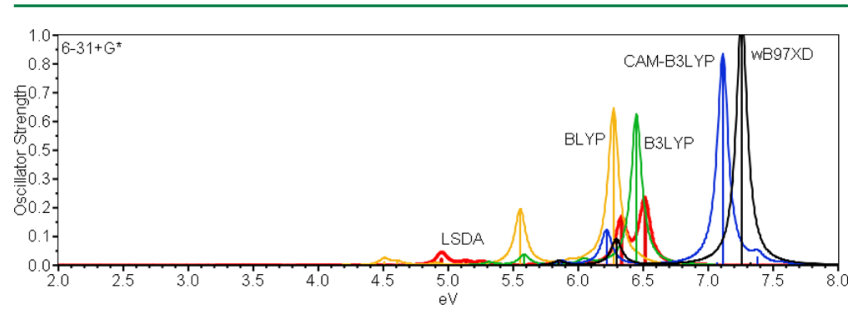

Figure 3. Effect of HF exchange on UV spectra of eclipsed (D5h) ferrocene.

first three dipole-forbidden states (see Table 1, invisible in Figure 3) differ very little with B3LYP, CAM-B3LYP, and wB97XD. Only BLYP and, in particular, LSDA predict substantially higher excitation energies for these states. With LSDA, this can be traced back to the short $\mathrm{Fe}-\mathrm{Cp}$ distance (1.579 A), which strongly influences low excitation energies. Note also that the first two states have almost the same energy with inversed order, compared to the other functionals. In addition, LSDA leads to a splitting of the main peak at $6.31 \mathrm{eV}$ into two weak states. A search for additional peaks, which included 70 excited stated states and covered the energy range up to $6.77 \mathrm{eV}$, produced no additional absorption. Thus, LSDA has trouble with the structure and with high and low energy peaks and will not be considered further.

Comparison of BLYP and B3LYP results reveals that HF exchange decreases low and increases high excitation energies, pushing the states apart. Range-separated hybrid functionals CAM-B3LYP and wB97XD continue this trend as low excitation energies increase by $0.15 \mathrm{eV}$, at the most, but the high energy peaks by up to $1 \mathrm{eV}$. As a result, range-separated functionals overestimate the excitation energy of the strongly allowed state of ferrocene severely, even with large basis sets (compare with Figure 2), while leading to no improvement for the low-energy states. This leaves us with BLYP and B3LYP. However, BLYP/6-31G* predicts a lower excitation energy for the strong peak than B3LYP and is therefore expected to underestimate the excitation energy upon basis set enlargement more than B3LYP, which already slightly below experiment. A further drawback of BLYP is that it produces a large number of spurious low-lying states. ${ }^{39}$ With the $6-31+G^{*}$ basis set, the strong state is excited state number 78 with BLYP, number 49 with B3LYP, number 46 with CAM-B3LYP, and number 42 with wB97XD. Thus, 36 states predicted with BLYP disappear with the range-separated $\mathrm{wB} 97 \mathrm{XD}$ functional that includes $100 \%$ of long-range HF exchange. A ZINDO calculation, producing the main absorption as excited state 43 at $6.85 \mathrm{eV}$, confirms that the remaining low-lying states are not DFT artifacts. With the aug-CC-PVTZ basis set, B3LYP and CAMB3LYP agree that the strong state is excited state number 51 . Thus, B3LYP with $20 \%$ of HF exchange removes the spurious states equally well as CAM-B3LYP.

Taking all issues together, the global hybrid B3LYP is the only one among the tested density functionals that can predict the spectrum of ferrocene over the entire range of excitation energies from $2 \mathrm{eV}$ to $7 \mathrm{eV}$. Therefore, B3LYP will be used for the following detailed analysis of the ferrocene spectrum.

The Visible Part of the Spectrum. The spectra presented so far do not show any absorption in the visible and consequently predict ferrocene to be colorless. However, calculations with all density functionals and basis sets produce a large number of excited states with zero oscillator strength below the strongly allowed transition. Figure 4 depicts TDB3LYP $/ 6-31+\mathrm{G}^{*}$ energies of all excited states, regardless of oscillator strength. (Peaks that have nonzero oscillator strength are shifted slightly upward.)

Figure 4 shows four isolated peaks at low energy; two of them are in the visible range (below $3.2 \mathrm{eV}$ ). Each peak consists

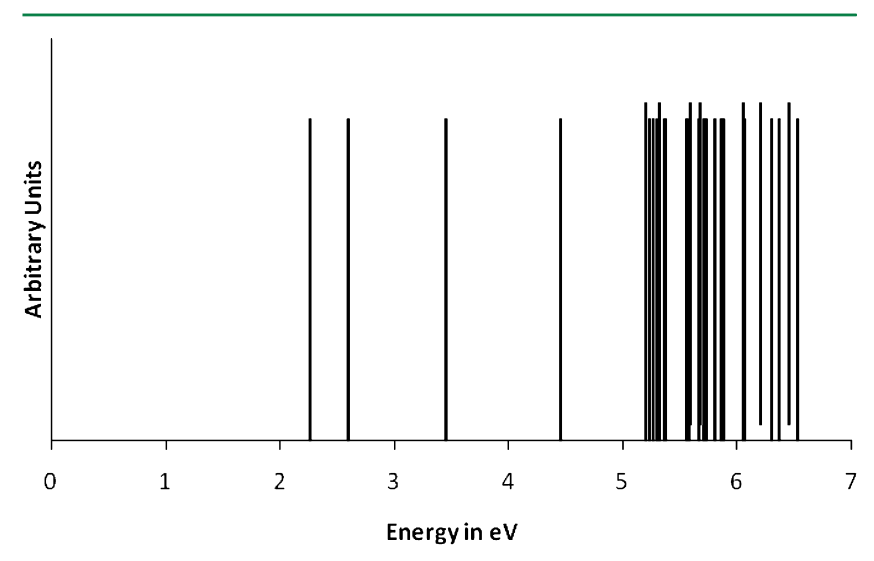

Figure 4. States of ferrocene below $6.5 \mathrm{eV}$ at B3LYP/6-31+G*. 
of two degenerate states. These four excited states in the visible part of the spectrum are dipole-forbidden but may become weakly allowed through vibronic coupling. ${ }^{8}$ Most dipoleforbidden states are found between 5 and $6 \mathrm{eV}$, where ferrocene starts to absorb more strongly. The effect of vibrations was modeled by calculating the IR spectrum of the D5h ground state and extracting vibrationally excited structures according to normal modes 1, 2 (degenerate with 3), 4, 5 (degenerate with 6), and 7. Then, UV spectra of the vibrationally excited structures (Scheme 1) were calculated and added to the spectrum of the D5h form in Figure 5. The inset shows the low-energy part of the spectrum with stretched vertical axes, to make the weak absorptions visible.

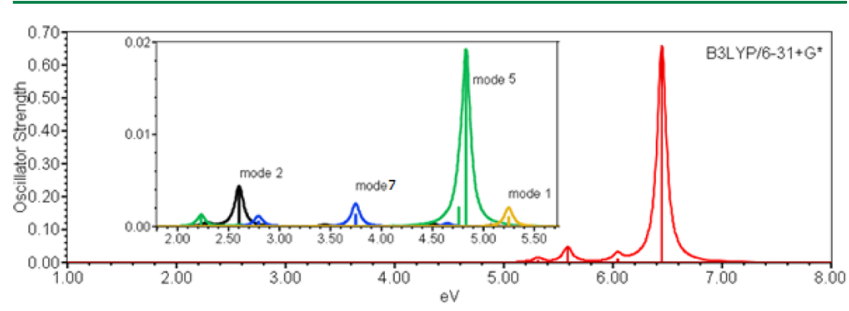

Figure 5. UV spectrum of ferrocene in vibrational ground and excited states at the B3LYP/6-31+G* level of theory.

Mode 1 at $43 \mathrm{~cm}^{-1}$ does not lead to absorption in the visible region. Mode $2\left(162 \mathrm{~cm}^{-1}\right)$ (black spectrum in Figure 5) activates excited states 3 and 6 (almost invisible). Normal mode 4 is a symmetric stretch of the $\mathrm{Cp}$ rings, relative to $\mathrm{Fe}$, and does not activate any excited states but shows that decreasing the $\mathrm{Fe}-\mathrm{Cp}$ distance by $0.2 \AA$ increases the first excitation energy from $2.2 \mathrm{eV}$ to $3.9 \mathrm{eV}$. Mode $5\left(363 \mathrm{~cm}^{-1}\right)$ activates excited state 2 at $2.24 \mathrm{eV}$ and excited state 10 at $4.83 \mathrm{eV}$. Mode 7 has its strongest effect on excited state 6, imparting oscillator strength on the peak at $3.75 \mathrm{eV}$. Energies and oscillator strength of the states activated by vibrations are given in Table 1 in italics below the corresponding states of the D5h form. It is interesting to note that the rather strong structural distortions (with exception of decreasing the $\mathrm{Fe}-\mathrm{Cp}$ distance) change the excitation energies of the states relatively little.

In Figure 5, the lower vibrational energy of mode 2, compared to mode 5, and the higher oscillator strength of excited state 3 (black), compared to excited state 2 (green), suggests that the experimentally observed peak at $2.82 \mathrm{eV}$ is excited state number 3 calculated at $2.61 \mathrm{eV}$ and not excited state 1 (degenerate with 2) as assumed in other theoretical studies. ${ }^{6,10,12,15}$ Excited state number 2 at $2.24 \mathrm{eV}$ seems to be responsible for the shoulder at $2.35 \mathrm{eV}$, which was suggested by Scott and Becker ${ }^{16}$ and by Scuppa et al. ${ }^{12}$ to arise from a triplet excitation. The assignment of excited state 2 to the $2.35 \mathrm{eV}$ shoulder, however, is in agreement with the disappearance of this peak and the color change of ferrocene from orange to yellow upon cooling. ${ }^{16}$ Because the vibrational energy of mode 5 is substantially higher than that of mode 2 , cooling should let excited state 2 at $2.24 \mathrm{eV}$ vanish before excited state 3 at 2.61 $\mathrm{eV}$. Since the absorbance at $2.24 \mathrm{eV}$ is in the green part of the spectrum with the complementary color red, ferrocene should appear more orange at higher temperature. Of course, the presence of this singlet state does not rule out the simultaneous presence of a triplet state. The weak absorption at $3.82 \mathrm{eV}$ in the experimental spectrum appears to be related to excited state number 6 at $3.75 \mathrm{eV}$, and the features at 4.68 and $4.77 \mathrm{eV}$ can be associated with strong absorption at $4.83 \mathrm{eV}$ produced by mode 5 .

Low-Lying Allowed States. The absorption spectrum of ferrocene vapor at $35{ }^{\circ} \mathrm{C}$ shows at least six peaks in the range between $4.5 \mathrm{eV}$ and $6.2 \mathrm{eV}$. The top panel of Figure 6 shows an
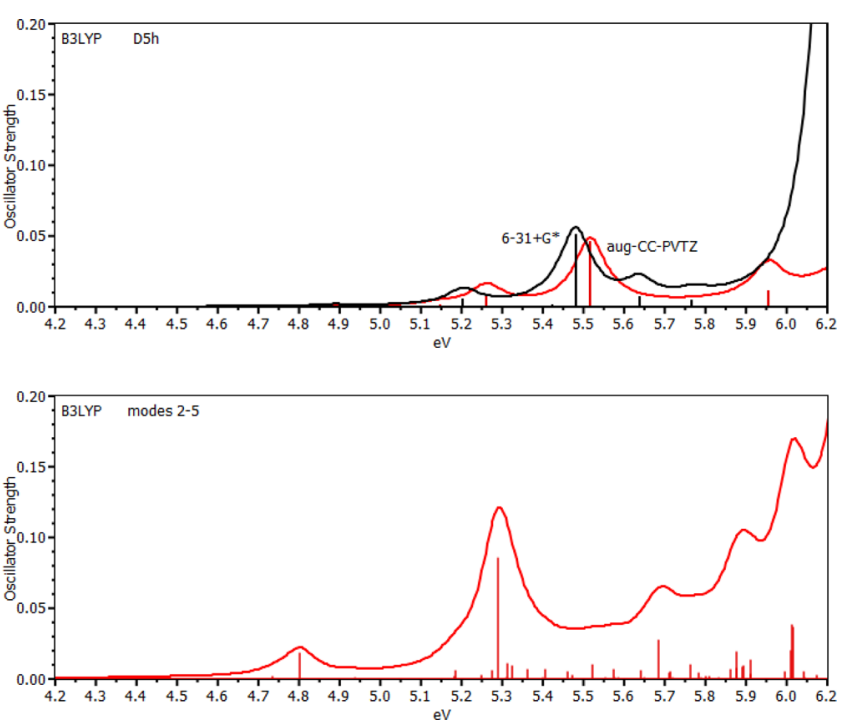

Figure 6. (Top) Allowed states of DSh ferrocene at B3LYP/6-31+G* and B3LYP/aug-CC-PVTZ. (Bottom) Combined spectra of ferrocene D5h and of vibrationally excited structures corresponding to modes 2 and 5 .

enlarged version of this spectral range, as predicted at the B3LYP/6-31+G* and B3LYP/aug-CC-PVTZ levels for the $\mathrm{D} 5 \mathrm{~h}$ form. The energies of the peaks differ by $<0.1 \mathrm{eV}$ with the two basis sets, and both basis sets predict two or three peaks but not six or more. There are many weakly allowed diffuse states in this energy range (the last two rows of Table 1). The positions of these states are dependent strongly on the amount of HF exchange and they move out of this energy range with range-separated functionals. As outlined above, it is reasonable to assume that these states are spurious CT states. However, there are many dipole-forbidden valence states in this energy range (see Figure 4) that persist with wB97XD and ZINDO. Therefore, it is reasonable to assume that the missing peaks are vibrationally excited states. The spectrum in the bottom panel of Figure 6 is a composition of the D5h spectrum plus spectra of vibrationally excited structures of modes 2 and 5. The spectrum shows a remarkable similarity with the vapor spectrum of ferrocene, reproducing all the major features of the vapor spectrum between 4 and $6 \mathrm{eV}$ with errors of $0.2 \mathrm{eV}$ or less. Although such an approach can only provide an estimate for the vibronically coupled absorption spectrum (no attempt was made for weighting the contribution according to vibrational energy for instance), the results present strong evidence that the visible region and the region between 4 and 6 $\mathrm{eV}$ of the ferrocene spectrum are strongly influenced by vibronic coupling.

Characterization of the Excited States. According to ligand field theory, the low-lying excited states of ferrocene are $\mathrm{d}-\mathrm{d}$ transitions on $\mathrm{Fe}$ and the higher energy states are CT states. To assess the actual amount of charge transfer upon electronic excitation, excited states contributing most to the spectrum are examined with density difference plots and NBO analysis. $^{22,40,41}$ 
The charge in the D5h ground state on $\mathrm{Fe}$ is +0.56 e at B3LYP/6-31G* but changes to -0.27 e at B3LYP/6-31+G*. This might come as a surprise, considering that the oxidation state of $\mathrm{Fe}$ is +2 . However, ferrocene has been shown, theoretically, to be $\sim 50 \%$ covalent, $^{42}$ and the result is in agreement with experimental findings by Makal et al. ${ }^{43} \mathrm{NBO}$ analysis is quite independent of the basis set and large shifts in NBO charges upon enlargement of the basis set, usually reflect true improvements in the electron density. ${ }^{44}$ This is consistent with the improvement of the spectra upon inclusion of diffuse functions. In any case, shifts in charge density upon excitation are qualitatively similar with $6-31 \mathrm{G}^{*}$ and $6-31+\mathrm{G}^{*}$ basis sets (albeit larger with $6-31+\mathrm{G}^{*}$, especially for more-diffuse highlying states).

The order of the occupied orbitals that are involved in the excitations is the same with all density functionals (excluding LSDA) and basis sets. Orbitals 42 and 43 are bonding linear combinations of Fe d- and Cp-HOMO orbitals of $4 \mathrm{e}_{1}$ " symmetry. Orbitals 44 and $45\left(6 \mathrm{e}_{1}{ }^{\prime}\right)$ are bonding combinations of Fe p- and Cp-HOMO orbitals. Orbital 46 is the Fe $\mathrm{d}_{z}{ }^{2}$ orbital of $8 \mathrm{a}_{1}{ }^{\prime}$ symmetry. The degenerate HOMO pair is the bonding combination of Fe d- and Cp-LUMO orbitals of $4 \mathrm{e}_{2}{ }^{\prime}$ symmetry. The ordering of the unoccupied orbitals depends on the basis set. With increasing number of diffuse functions, more lowlying diffuse orbitals appear between the valence orbitals. The strongly allowed excited states predicted with different basis sets, however, involve analogous orbitals, regardless how many diffuse functions have slipped between them. Only the number of states with little or no oscillator strength differs as described in the previous section. The following discussion is done with $6-31+G^{*}$ for the low-lying states where there is little difference with different basis sets and with $6-31+\mathrm{G}^{*}$ and aug-CC-PVTZ basis sets for the high-energy part of the spectrum.

Excited states 1 and 2 (Figure 7, top) are electronic transitions from the two degenerate HOMOs $\left(4 \mathrm{e}_{2}{ }^{\prime}\right)$ to the two degenerate $\left(5 \mathrm{e}_{1}{ }^{\prime \prime}\right)$ unoccupied molecular orbitals. The $4 \mathrm{e}_{2}{ }^{\prime}$ orbitals are polarized toward $\mathrm{Fe}$, the $\mathrm{Se}_{1}{ }^{\prime \prime}$ orbitals toward $\mathrm{Cp}$. Accordingly, the density difference plots reveal increased electron density (light blue) on $\mathrm{Cp}$ and decreased electron
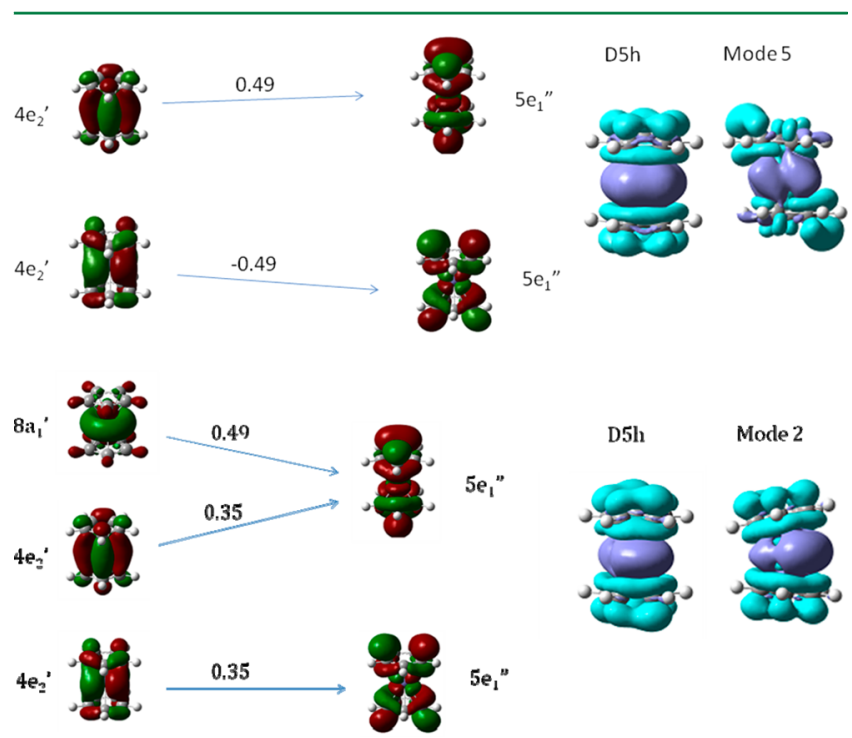

Figure 7. (Top) Excited state 2, $2.26 \mathrm{eV} f=0$ in the D5h form, 2.24 $\mathrm{eV}, f=0.0012$ in mode 5. (Bottom) Excited state 3, $2.60 \mathrm{eV}, f=0$ in the D5h form, $2.61 \mathrm{eV}, f=0.0044$ in mode 2 . density (dark blue) on $\mathrm{Fe}$ in the excited state. The amount of charge transferred is $0.40 \mathrm{e}$, according to NBO analysis.

Excited states 3 and 4 (see Figure 7, bottom panel) additionally involve the $\left(8 \mathrm{a}_{1}{ }^{\prime}\right) \mathrm{d}_{z^{2}}$-orbital of $\mathrm{Fe}$ but are otherwise very similar to states 1 and 2 . Similar to the previous case, $\mathrm{Cp}$ rings act as electron acceptors. The amount of charge transferred from $\mathrm{Fe}$ to $\mathrm{Cp}$ is also $0.40 \mathrm{e}$.

Excited states 5 and 6 (see Figure 8) are different linear combinations of the same orbitals involved in excited states 3 and 4 . The amount of charge transfer is $0.41 \mathrm{e}$.

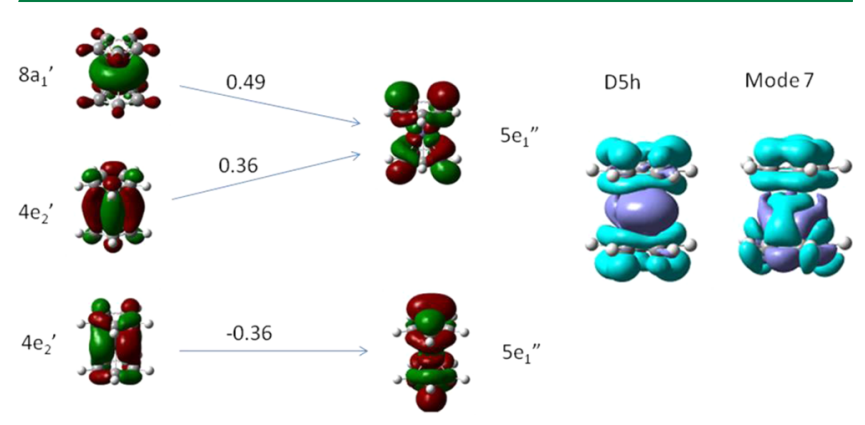

Figure 8. Excited state 6, $3.45 \mathrm{eV}, f=0$ in the $\mathrm{D} 5 \mathrm{~h}$ form, $3.75 \mathrm{eV}, f=$ 0.0012 in mode 7.

Excited state number 14 (Figure 9) arises from an electronic transition from $6 \mathrm{e}_{1}{ }^{\prime}$ to $5 \mathrm{e}_{1}{ }^{\prime \prime}$. The total charge transfer amounts to $0.38 \mathrm{e}$.

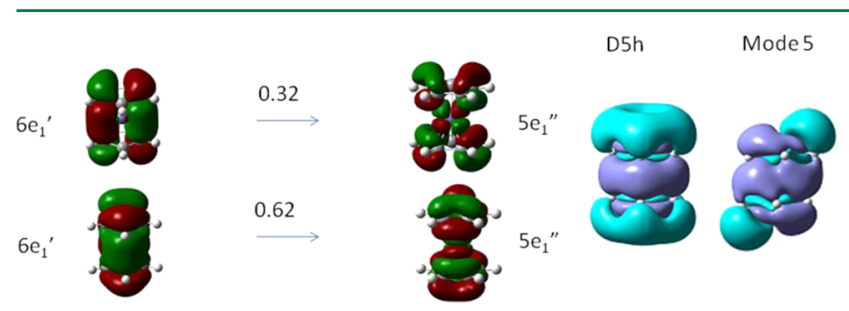

Figure 9. Excited state 14, $5.26 \mathrm{eV}, f=0$ in the D $5 \mathrm{~h}$ form, $4.83 \mathrm{eV}, f=$ 0.0186 in mode 5 .

The first allowed states of the D5h form are excited states 18 and 19 (see Figure 10) which involve transitions from $4 \mathrm{e}_{2}^{\prime}$ to a combination of higher-lying unoccupied valence and diffuse orbitals of $5 \mathrm{e}_{2}{ }^{\prime}$ and $6 \mathrm{e}_{2}{ }^{\prime}$ symmetry with a total charge transfer of

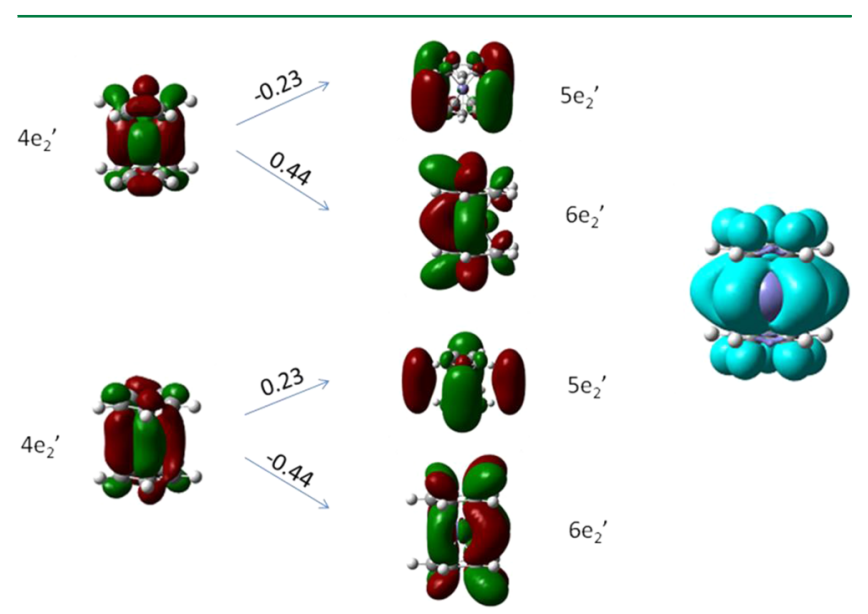

Figure 10. Excited state 18, $5.32 \mathrm{eV}, f=0071$, D5h. 
0.70 e. These states are the only ones with a substantially larger charge transfer.

The next allowed state of the DSh form is excited state number 26 at $5.59 \mathrm{eV}$ (see Figure 11). This state arises from

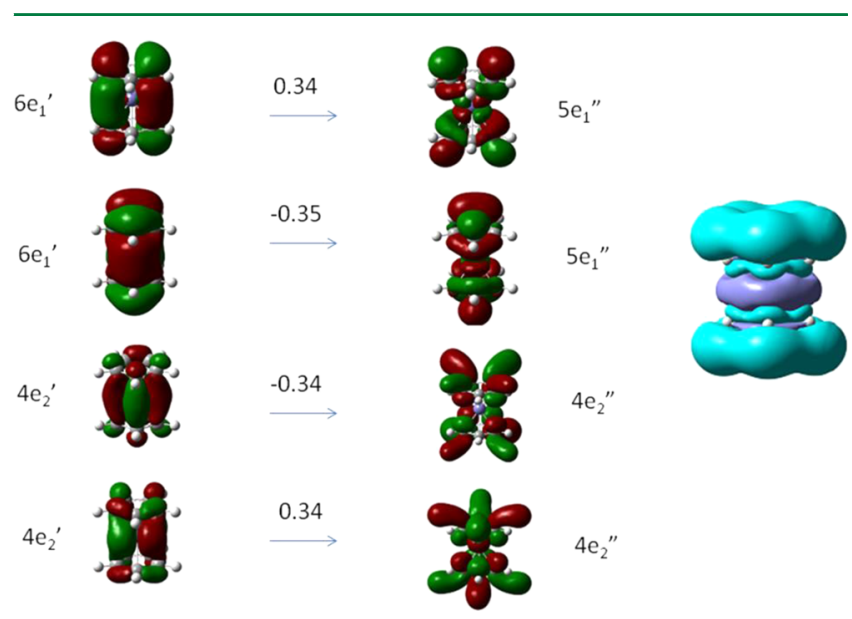

Figure 11. Excited state 26, $5.59 \mathrm{eV}, f=0.0459$, D5h.

electron transfer from a linear combination of $4 \mathrm{e}_{2}{ }^{\prime}$ with the $6 \mathrm{e}_{1}{ }^{\prime}$ orbitals below. The acceptors are $5 \mathrm{e}_{1}{ }^{\prime \prime}$ and $4 \mathrm{e}_{2}{ }^{\prime \prime}$ orbitals, the latter being the antibonding combination of the Cp-LUMOs having no coefficient on Fe. The amount of charge transfer is 0.30 e.

The main peak of the spectrum (see Figure 12) involves the same orbitals as excited state 26 in Figure 11. The density

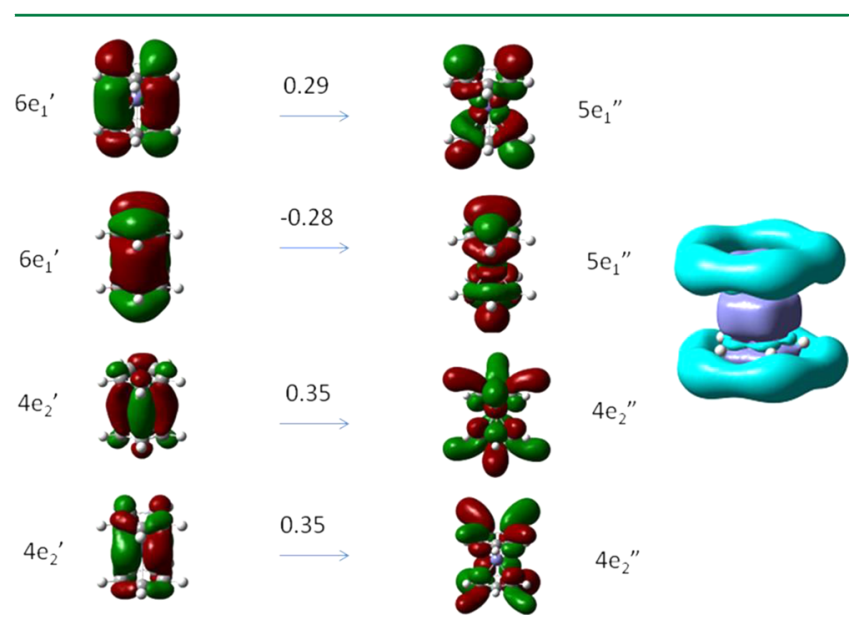

Figure 12. Excited state 49, $6.45 \mathrm{eV}, f=0.66$, D5h.

difference plot reveals the diffuse nature of this excited state and rationalizes the large influence of diffuse functional on its excitation energy. Nonetheless, the CT amounts to only $0.46 \mathrm{e}$. The splitting of this state into a main peak and a lower energy satellite (excited state 26) is obtained at all levels of theory employed in this investigation. The satellite falls into the region where ferrocene starts absorbing and is mixed with the vibrationally coupled dipole forbidden transitions.

Because $6-31+\mathrm{G}^{*}$ and aug-CC-PVTZ results predict very different oscillator strengths for the main peak of the spectrum, orbital plots of the main excitation and the two following states are shown also at B3LYP/aug-CC-PVTZ. Figure 13 shows no dramatic difference between the main peaks with the two basis sets. With aug-CC-PVTZ there are two very small additional

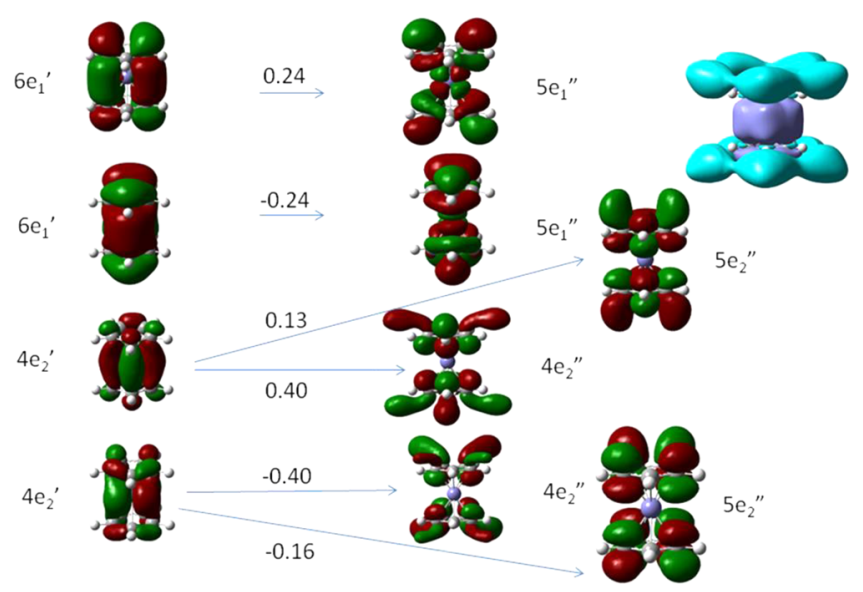

Figure 13. Excited state 51, with B3LYP/aug-CC-PVTZ, $6.20 \mathrm{eV}, f=$ 0.34 , D5h.

electronic configurations from the HOMOs into two additional higher-lying Cp antibonding orbitals of $\mathrm{e}_{2}{ }^{\prime \prime}$ symmetry.

Excited state 80 at $6.73 \mathrm{eV}$ (see Figure 14) involves linear combinations of the $4 \mathrm{e}_{1}{ }^{\prime \prime}$ and $4 \mathrm{e}_{2}{ }^{\prime}$ orbitals with contributions from $6 \mathrm{e}_{1}{ }^{\prime}$ and $8 \mathrm{a}_{1}{ }^{\prime}$ and transfers electrons into $5 \mathrm{e}_{1}{ }^{\prime \prime}$ and $5 \mathrm{e}_{2}{ }^{\prime \prime}$ orbitals and into diffuse functions.

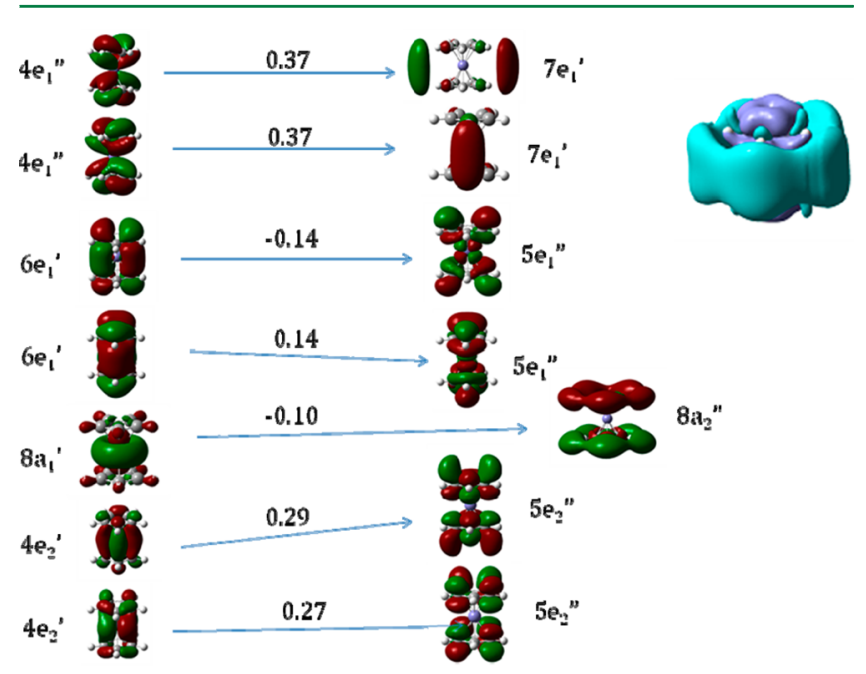

Figure 14. Excited state 80, with B3LYP/aug-CC-PVTZ, $6.73 \mathrm{eV}, f=$ 0.15 , D5h.

Excited state 85 (see Figure 15) resembles excited states 51 ( 49 with $6-31+\mathrm{G}^{*}$ ) and the satellite state 26 (with $6-31+\mathrm{G}^{*}$ ). As excited states 51, 80, 85, and the satellite involve the same donor orbitals, the oscillator strength is distributed among these states. ${ }^{35-37}$ As stated previously, the splitting is absent with CAM-B3LYP and might be an artifact of the diffuse but finite basis set that might lead to artificially bound states that are actually above the ionization threshold.

The molecular orbitals in Figures $7-15$ reflect the partially covalent ${ }^{42}$ nature of ferrocene, since all occupied orbitals are delocalized over the entire molecule. In all excited states, the $\mathrm{Cp}$ rings act as electron acceptors, so that none of the excited states can be considered a pure $\mathrm{d}-\mathrm{d}$ transition. The weak formally forbidden absorptions at 2.24 and $2.61 \mathrm{eV}$ shift only 0.06 e less from $\mathrm{Fe}$ to $\mathrm{Cp}$ than the strong absorption at $6.45 \mathrm{eV}$, which is generally assumed to be a CT state. The only state that sticks out is excited state 18 , which has a charge transfer of 0.70 


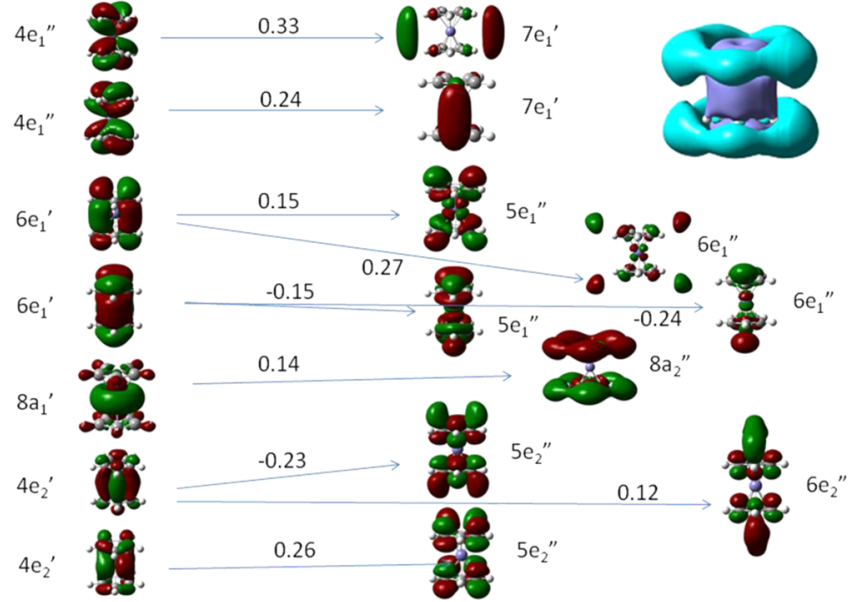

Figure 15. Excited state 85, with B3LYP/aug-CC-PVTZ, $6.79 \mathrm{eV}, f=$ 0.27 , D5h.

e. The distinction between the so-called $d-d$ transitions and the CT states of ferrocene is therefore unjustified.

Comparison with Earlier Work. Table 2 summarizes relevant results from previous theoretical studies, leading to the conclusion that TDB3LYP should not be used to calculate the absorption spectrum of ferrocene. Boulet et al. ${ }^{6}$ employed timedependent density functional response theory (TDDFRT) with LDA and gradient corrected functionals. The basis sets consisted of pseudo-potentials plus Slater-type polarized triple- $\zeta$ valence basis sets. The results were compared to those at TDB3LYP/LAND2DZ. TDB3LYP faired particularly poorly, in comparison with LB94/LDA results. Boulet et al. considered the LDA structure superior to the B88P86 structure, because the former leads to $0.3 \mathrm{eV}$ higher excitation energies. In contrast, the LDA structure is clearly inferior because the $\mathrm{Fe}-$ $\mathrm{Cp}$ distance is too short. Table 2 shows that the first excitation energy at TDB3LYP/LAND2DZ is $0.44 \mathrm{eV}$ below that obtained here with large all-electron basis sets. Thus, the difference between TDB3LYP and TDDFRT results stems largely from use of different geometries and different basis sets. Finally, underestimation of the excitation, compared to the experiment, is aggravated by assigning the first rather than the third excited state to the absorption at $2.82 \mathrm{eV}$.

Scuppa et al. ${ }^{12}$ included relativistic effects through the zeroorder regular approximation (ZORA) and spin orbit effects with the van Leeuwen-Baerends (LB94) correlation functional and concluded that spin-orbit effects are small. The best results were found with the PBE geometry that predicts a $\mathrm{Cp}-\mathrm{Fe}$ distance 1.638 Å. As shown already by Boulet, decreasing the
$\mathrm{Cp}-\mathrm{Fe}$ distance increases the first excitation energy. Therefore, using an incorrect geometry is not an improvement but rather error cancellation.

Comparison of the results of Ishimura et al. ${ }^{7}$ employing the symmetry-adapted cluster configuration interaction (SAC-CI) method with the present ones shows several differences between the SAC-CI and DFT methods. First, the ordering of the HFT orbitals, which are the starting point for the SACCI calculations, is very different from that of DFT orbitals. Previous work on HFT and DFT orbital energies, including inner valence down to $-30 \mathrm{eV}$, suggests that the DFT orbital ordering and energy spacing are usually in much better agreement with the experiment than HFT orbital energies. ${ }^{45,46}$ Because of the different orbital orderings, the excited states with SAC-CI and DFT also differ. With SAC-CI, there is not a single excited state involving the low-lying unoccupied $5 \mathrm{e}_{1}{ }^{\prime \prime}$ orbital. Instead, $9 \mathrm{e}_{1}{ }^{\prime \prime}$, which lies almost $7 \mathrm{eV}$ above $5 \mathrm{e}_{1}{ }^{\prime \prime}$, is the preferred acceptor. Nonetheless, the energies and electronic configurations of the first three low lying states with SAC-CI are in agreement with TDB3LYP (although the $4 \mathrm{e}_{2}{ }^{\prime}-5 \mathrm{e}_{1}{ }^{\prime \prime}$ (HOMO-LUMO) transition at TDB3LYP corresponds to the $4 \mathrm{e}_{2}{ }^{\prime}-9 \mathrm{e}_{1}{ }^{\prime \prime}(\mathrm{HOMO}-2$ to LUMO+6) transition with SAC-CI). The first excited state that is generally claimed to be underestimated at the TDB3LYP level occurs at even lower energy with the $a b$ initio method. For the states between 5.25 $\mathrm{eV}$ and $5.60 \mathrm{eV}$, originating from the $4 \mathrm{e}_{1}{ }^{\prime \prime}$ orbitals, there is no counterpart with DFT, since all states in this energy range arise from $6 \mathrm{e}_{1}^{\prime}$ and $4 \mathrm{e}_{2}{ }^{\prime}$ orbitals. The first allowed peak, which Ishimura et al. assigned to the strong absorption of ferrocene, is a transition into two diffuse orbitals of $\mathrm{a}_{1}{ }^{\prime}$ symmetry. Ishimura et al. do not give the oscillator strength for any of the states, but the present work shows that excitations into the diffuse states have very low oscillator strength. Therefore, it seems more likely that the strong state is the one at $6.45 \mathrm{eV}$, which arises from transfer of electrons from $6 \mathrm{e}_{1}{ }^{\prime}$ to $9 \mathrm{e}_{1}{ }^{\prime \prime}$. This state is similar in nature to the strong state at TDB3LYP, which involves $6 \mathrm{e}_{1}{ }^{\prime}$ to $5 \mathrm{e}_{1}{ }^{\prime \prime}$.

The TDB3LYP results of Li et al. ${ }^{10}$ differ by less than $0.1 \mathrm{eV}$ from the present ones. $\mathrm{Li}$ et al. calculated only the first four excited states, which are predicted at slightly higher energy with pure DFT functionals than with hybrids. The conclusion that PBE should be preferred over B3LYP arises from assigning excited state 1 rather than excited state 3 to the peak at $2.82 \mathrm{eV}$.

Finally, Fromager ${ }^{15}$ compared TDB3LYP and TDCAMB3LYP excitation energies with the relativistic version of the basis set employed here and concluded that range-separated functionals should be used for metallocenes. The low excitation energies are very similar with both functionals and with the present results. This confirms that the relativistic effects are

Table 2. Comparison of Present Results with Those of Previous Work

\begin{tabular}{|c|c|c|c|c|c|c|}
\hline \multicolumn{7}{|c|}{ Excitation Energy $(\mathrm{eV})$} \\
\hline this work B3LYP/aug-CC-PVTZ & Boulet $^{6}$ & Ishimura $^{7} \mathrm{SAC}-\mathrm{CI}$ & $\mathrm{Li}^{10} \mathrm{~B} 3 \mathrm{LYP}$ & Scuppa $^{12}$ ZORA & Fromager ${ }^{15}$ B3LYP & CAM-B3LYP \\
\hline 2.18 & 1.74 & 2.11 & 2.18 & 2.81 & 2.43 & 2.33 \\
\hline 2.51 & 2.27 & 2.27 & 2.53 & 2.91 & 2.70 & 2.65 \\
\hline 3.45 & 3.22 & 4.03 & 3.38 & 3.44 & 3.48 & 3.45 \\
\hline 5.26 & & $5.29^{a_{*}}$ & & & 5.41 & 5.85 \\
\hline 5.55 & & $5.48^{a}$ & & & 5.72 & 6.25 \\
\hline 5.89 & & 6.34 & & & & \\
\hline 6.20 & & 6.45 & & & & \\
\hline
\end{tabular}

${ }^{a}$ These states are similar in energy to the DFT states but arise from different electronic transitions. 
small for ferrocene and that a nonrelativistic all-electron basis sets can be employed. The oscillator strength of the two first allowed states with the B3LYP functional at 5.41 and $5.72 \mathrm{eV}$ (here, $5.26 \mathrm{eV}(0.0054)$ and $5.55 \mathrm{eV}(0.0514)$ ) were omitted, giving the impression that there are no allowed states in this region with B3LYP. Moreover, Fromager et al. erroneously assigned the excited state at $6.25 \mathrm{eV}$ with an oscillator strength of 0.107 to the strong absorption at $6.12 \mathrm{eV}$ and concluded that CAM-B3LYP predicts correct excitation energy but underestimates oscillator strength. It is obvious from a comparison with data in Table 1 and Figure 2 that the state at $6.25 \mathrm{eV}$ is the low-energy satellite of the strongly allowed state that is shifted to higher energy with CAM-B3LYP, compared to B3LYP. It is typical for range-separated functionals to predict higher excitation energies than global hybrids also for conjugated $\pi$ systems. ${ }^{47}$ Because the overestimated energy of this satellite almost coincides with the strong transition found experimentally at $6.12 \mathrm{eV}$, Fromager et al. missed the proper state which is predicted at $6.99 \mathrm{eV}$ with an oscillator strength of 0.58 at CAMB3LYP/aug-CC-PVTZ. Therefore, the correct conclusion is that CAM-B3LYP overestimates the excitation energy, while the oscillator strength is reasonable.

\section{CONCLUSIONS}

The structure and absorption spectrum of ferrocene can be calculated with errors not exceeding $0.2 \mathrm{eV}$ over the entire range of excitation energies at the TDB3LYP level of theory with all-electron basis sets that include diffuse functions. Relativistic effects are negligible. Earlier claims regarding the failure of TDB3LYP are demonstrated to arise from use of the inadequate LANLDZ pseudo-potential basis sets and incorrect assignment of the peaks.

Dipole-forbidden excited states of ferrocene in the visible range can be modeled by using vibrationally excited structures. Energies and oscillator strengths are in good agreement with experiment and explain the color change from orange to yellow of ferrocene upon cooling.

Low and high energy excitations involve almost identical amounts of charge transfer (0.40-0.46 e), according to NBO analysis. Therefore, the differentiation into Fe-centered $d-d$ transitions and CT states is not borne out by the present calculations. The only state with a larger charge transfer $(0.70$ e) is excited state 18 at $5.32 \mathrm{eV}$.

The effect of including increasing amounts of Hartree-Fock (HF) exchange into the density functional is a reduction in low excitation energies and an increase in high excitation energies. Examination of the entire spectrum suggests that B3LYP is once more the golden middle. Twenty percent $(20 \%)$ of HF exchange with B3LYP is sufficient to remove most of the 36 spurious charge-transfer states that are present with BLYP/6$31+\mathrm{G}^{*}$. In contrast, large amounts of HF exchange in the longrange part of the functional, as with CAM-B3LYP and wB97XD, are excessive and lead to overestimation of energies of diffuse states, such as the strong absorption at 6.31 by $0.8 \mathrm{eV}$.

\section{AUTHOR INFORMATION}

\section{Corresponding Author}

*Tel.: +90 312290 2122. Fax: +90 312266 4068. E-mail: salzner@fen.bilkent.edu.tr.

\section{Notes}

The authors declare no competing financial interest.

\section{ACKNOWLEDGMENTS}

This work was supported by Bilkent University. The author thanks Lutfiye Hallioglu, Eylul Karaca, and Cansu Kavsat for doing some of the calculations during their undergraduate projects. The author thanks Prof. Michael Bendikov and Prof. Roi Baer for helpful discussions.

\section{DEDICATION}

This work is dedicated to the memory of my dear friend and colleague, Prof. Michael Bendikov of the Weizmann Institute of Science, who passed away July 2, 2013.

\section{REFERENCES}

(1) Kealy, T. J.; Pauson, P. L. Nature 1951, 168, 1039-1040.

(2) Huheey, J. E.; Keiter, E. A.; Keiter, R. L. Inorganic Chemistry; 4th ed. ed.; Harper Collins: New York, 1993.

(3) Kirchner, R.; Loew, G.; Mueller-Westerhoff, U. Theor. Chim. Acta 1976, 41, 1-6.

(4) Taylor, T. E.; Hall, M. B. Chem. Phys. Lett. 1985, 114, 338-342.

(5) Koch, H.; Jorgensen, P.; Helgaker, T. J. Chem. Phys. 1996, 104, 9528-9530.

(6) Boulet, P.; Chermette, H.; Daul, C.; Gilardoni, F.; Rogemond, F.; Weber, J.; Zuber, G. J. Phys. Chem. A 2001, 105, 885-894.

(7) Ishimura, K.; Hada, M.; Nakatsuji, H. J. Chem. Phys. 2002, 117, 6533.

(8) Parac, M.; Grimme, S. J. Phys. Chem. A 2002, 106, 6844-6850.

(9) Nemykin, V. N.; Makarova, E. A.; Grosland, J. O.; Hadt, R. G.; Koposov, A. Y. Inorg. Chem. 2007, 46, 9591-9601.

(10) Li, Y. L.; Han, L.; Mei, Y.; Zhang, J. Z. H. Chem. Phys. Lett. 2009, 482, 217-222.

(11) Martin, J.; Baker, J.; Pulay, P. J. Comput. Chem. 2009, 30, 881883.

(12) Scuppa, S.; Orian, L.; Dini, D.; Santi, S.; Meneghetti, M. J. Phys. Chem. A 2009, 113, 9286-9294.

(13) Bean, D. E.; Fowler, P. W.; Morris, M. J. J. Organomet. Chem. 2011, 696, 2093-2100.

(14) Mohammadi, N.; Ganesan, A.; Chantler, C. T.; Wang, F. J. Organomet. Chem. 2012, 713, 51-59.

(15) Fromager, E.; Knecht, S.; Jensen, H. J. A. J. Chem. Phys. 2013, 138, 084101-1-084101-14.

(16) Scott, D. R; Becker, R. S. J. Chem. Phys. 1961, 35, 516-531.

(17) Scott, D. R.; Becker, R. S. J. Chem. Phys. 1961, 35, 2246-2247.

(18) Armstrong, A. T.; Smith, F.; Elder, E.; McGlynn, S. P. J. Chem. Phys. 1967, 46, 4321-4328.

(19) Sohn, Y. S.; Hendrickson, D. N.; Hart Smith, J.; Gray, H. B. Chem. Phys. Lett. 1970, 6, 499-501.

(20) Coriani, S.; Haaland, A.; Helgaker, T.; Jorgensen, P. Chem. Phys. Chem. 2006, 7, 245-249.

(21) Yamaguchi, Y.; Ding, W.; Sanderson, C. T.; Borden, M. L.; Morgan, M. J.; Kutal, C. Coord. Chem. Rev. 2007, 251, 515-524.

(22) Reed, E. A.; Curtiss, L. A.; Weinhold, F. Chem. Rev. 1988, 88, 899.

(23) Slater, J. C. In Quantum Theory of Molecular and Solids; McGraw-Hill: New York, 1974; Vol. 4.

(24) Vosko, S. H.; Wilk, L.; Nusair, M. Can. J. Phys. 1980, 58, 12001211.

(25) Becke, A. D. Phys. Rev. A 1988, 38, 3098-3100.

(26) Lee, C.; Yang, W.; Parr, R. G. Phys. Rev. B 1988, 37, 785-789.

(27) Becke, A. D. J. Chem. Phys. 1993, 98, 5648-5652.

(28) Yanai, T.; Tew, D. P.; Handy, N. C. Chem. Phys. Lett. 2004, 393, $51-57$.

(29) Chai, J.-D.; Head-Gordon, M. Phys. Chem. Chem. Phys. 2008, 10, $6615-6620$

(30) Dunning, J. T. H. J. Chem. Phys. 1989, 90, 1007-1023.

(31) Kendall, R. A.; Dunning, J. T. H.; Harrison, R. J. J. Chem. Phys. 1992, 96, 6796-6806. 
(32) Frisch, E.; Frisch, M. J.; Clemente, F. R.; Trucks, G. W. Gaussian 09 User's Reference; Gaussian, Inc.: Wallingford, CT, 2009.

(33) Frisch, M. J.; Trucks, G. W.; Schlegel, H. B.; Scuseria, G. E.; Robb, M. A.; Cheeseman, J. R.; Scalmani, G.; Barone, V.; Mennucci, B.; Petersson, G. A.; Nakatsuji, H.; Caricato, M.; Hratchian, H. P.; Izmaylov, A. F.; Bloino, J.; Zheng, G.; Sonneberg, J. L.; Hada, M.; Ehara, M.; Toyota, K.; Fukuda, R.; Hasegawa, J.; Ishida, M.; Nakajima, T.; Honda, Y.; Kitao, O.; Nakai, H.; Vreven, T.; Montgomery, J. A., Jr.; Peralta, J. E.; Ogliaro, F.; Bearpark, M.; Heyd, J. J.; Brothers, E.; Kudin, K. N.; Staroverov, V. N.; Kobayashi, R.; Normand, J.; Raghavachari, K.; Rendell, A.; Burant, J. C.; Iyengar, S. S.; Tomasi, J.; Cossi, M.; Rega, N.; Millam, J. M.; Klene, M.; Knox, J. E.; Cross, J. B.; Bakken, V.; Adamo, C.; Jaramillo, J.; Gomperts, R.; Stratmann, R. E.; Yazyev, O.; Austin, A. J.; Cammi, R.; Pomelli, C.; Ochterski, J. W.; Martin, R. L.; Morokuma, K.; Zakrzewski, V. G.; Voth, G. A.; Salvador, P.; Dannenberg, J. J.; Dapprich, S.; Daniels, A. D.; Farkas, O.; Foresman, J. B.; Ortiz, J. V.; Cioslowski, J.; Fox, D. J.; Gaussian, Inc.: Wallingford, CT, 2009.

(34) Allouche, A.-R. Gabedit, Version 2.1.8; 2007.

(35) Thomas, W. Naturwissenschaften 1925, 13, 627.

(36) Reiche, F.; Thomas, W. Z. Phys. 1925, 34, 510-525.

(37) Kuhn, W. Z. Phys. 1925, 33, 408-412.

(38) http://webbook.nist.gov/cgi/cbook.cgi?ID=C102545\&Mask= 20\#Ion-Energetics

(39) Magyar, R. J.; Tretiak, S. J. Chem. Theor. Comput. 2007, 3, 976987.

(40) Reed, A. E.; Weinhold, F. J. Chem. Phys. 1985, 83, 1736.

(41) Reed, E. A.; Weinstock, R. B.; Weinhold, F. J. Chem. Phys. 1985, 83, 735 .

(42) Rayón, V. M.; Frenking, G. Organomet. 2003, 22, 3304-3308.

(43) Makal, A. M.; Plazuk, D.; Zakrzewski, J.; Misterkiewicz, B.; Wozniak, K. Inorg. Chem. 2010, 49, 4046-4059.

(44) Reed, A. E.; Curtiss, L. A.; Weinhold, F. Chem. Rev. 1988, 88, 899.

(45) Salzner, U. J. Phys. Chem. A 2010, 114, 10997-11007.

(46) Salzner, U.; Baer, R. J. Chem. Phys. 2009, 131, 231101-1231101-4.

(47) Salzner, U.; Aydin, A. J. Chem. Theor. Comput. 2011, 7, 25682583. 